\title{
Synthesis and Analgesic Potential of 4-[4-chloro-3- (trifluoromethyl)-phenyl]-4-piperidinol analogues Against Pain Model in Mice
}

\author{
Shazia Haider \\ University of Karachi \\ Zafar Saify \\ University of Karachi \\ Nousheen Mushtaq \\ University of Karachi \\ Faheema Siddiqui \\ University of Karachi \\ Toqeer Rao \\ Federal Urdu University of Arts Sciences and Technology \\ Tabinda Mallick \\ University of Karachi \\ Nasreen Begum ( $\sim$ alinasreen29@yahoo.com ) \\ University of Karachi HEJ Research Institute of Chemistry
}

\section{Research Article}

Keywords: Analgesic activity, hot plate method, morphine, acetophenone, potent, efficacy, pain

Posted Date: August 5th, 2021

DOI: https://doi.org/10.21203/rs.3.rs-766239/v1

License: (c) (i) This work is licensed under a Creative Commons Attribution 4.0 International License. Read Full License

Version of Record: A version of this preprint was published at Medicinal Chemistry Research on February 24th, 2022. See the published version at https://doi.org/10.1007/s00044-022-02860-8. 


\section{Abstract}

In the study of designing pharmacophore models for analgesic, a series of 4-[4-chloro-3- (trifluoromethyl)-phenyl]-4-piperidinol (TFMP) derivatives were synthesized and characterized by physical and spectral method (HR-EIMS, HR-FABMS, ${ }^{1} \mathrm{H}-\mathrm{NMR},{ }^{13} \mathrm{C}-$ NMR, UV, and FT-IR). The analgesic action of the synthesized derivatives was estimated by means of Hot Plate Method. Most of the compounds displayed potent analgesic efficacy and an ultrashort to long duration of action. The results indicate that these compounds are useful as analgesics. Qualitatively nine compounds resemble morphine in pharmacological action, whereas three derivatives are devoid of any significant analgesic potential. In conclusion, among active compounds 3 (188\%), 5 (137\%), 6 (162\%), and 8 (107\%) respectively emerged as most effective analgesic and they depressed peripheral and centrally mediated pain by opioid dependent and independent systems.

\section{Introduction}

Pain is a complex body response to noxious stimulus both chemical (acetic acid and formalin) or physical (heat and cold pressure,) or chemical (acetic acid and formalin) [1, 2]. Primarily inflammation or tissue injuries have been connected with pain stimulation through the release of quite a lot of inflammatory mediators that sensitizes as well as amplify nociceptive responses [3]. Pain is together a major economic and clinical delinquent, distressing large population than diabetes, cancer and heart malady. Currently, variety of prescribed medicines are existing for managing of pain, opioid drugs, exclusively those substituted $\mu$-opioid receptor and associated ways. They have established to be the utmost active, regardless of more or less severe side effects comprising addiction, dependence, respiratory depression, pruritus, nausea, hypotension, and constipation. As a result it is domineering to develop novel $\mu$ opioid receptor analgesics that preserve their opioid analgesic properties but less or no adversative side effects [4-6].

Opioid drugs, epitomized by morphine, yield their pharmacological activities, plus analgesia, by interim on neuronal cell membranes receptors. In CNS opioid receptors have three main classes namely $\mu, \delta$ and $\kappa$. Later research directed the isolation and structure of the endogenous ligand of the ORL-1 receptor (opioid receptor-like receptor). Discovery of the new receptor provides chance in drug innovation for new compounds which can be administered as analgesic or additional syndromes moderated by this receptor [7-9]. As a consequence searches for other substitutes appear essential and favorable to get novel analgesic agents. Hot plate and tail immersion procedure are well known to be choosy for opioid like compounds in many animal species $[5,10]$. Piperidine ring being the part of morphine are recognized as powerful analgesic agents. More advanced investigation has been completed for the substituted piperidine derivatives to develop superior molecules for the treatment of pain [11-16]. Lots of efforts have been made to investigate potent analgesic agents without undesirable side effects in which piperidine moiety played important role [17-22]. Valuation of novel chemical compounds as analgesic agents by using animal models is a particularly complex assignment. In finding analgesic agents, researchers have developed a relationship between animal models and human clinical responses. These methods are simple, reproducible and sensitive to discover weak and potent analgesic agents.

Despite of all the researches and work which is already done on piperidine, still there is need to explore these types of molecules for analgesic potential with safe efficacy. In proposed study different derivatives of 4-[4-chloro-3-(trifluoromethyl)phenyl]-4-piperidinol (TFMP) was synthesized and evaluated for in vivo analgesic potential. The compounds being synthesized will be added to the current information of piperidine substituted compounds in the development of drugs.

\section{Results And Discussion}

\subsection{Synthesis}

We report here the synthesis of 4-[4-chloro-3- (trifluoromethyl)-phenyl]-4-piperidinol (TFMP) derivatives 1-12 via the reactions of TFMP by nucleophilic substitution with a variety of alkylating agent by $\mathrm{N}$-alkylation. We selected different aromatic acetophenone, acetonaphthone, oxane and oxalane and phenythyl bromide with the purpose of study the consequence of substituents on the piperidine ring of parent molecule (Scheme-1). To the best of our information, all compounds are reported 
here for the first time. The structures of synthesized compounds 1-12 were established by different physical and spectral method (HR-EI-MS, HR-FABMS, ${ }^{1} \mathrm{H}-\mathrm{NMR},{ }^{13} \mathrm{C}-\mathrm{NMR}, \mathrm{UV}$, and FT-IR) (Table-1).

\subsection{Analgesic Activity}

Hot plate methodology was utilized to investigate the analgesic potential of the synthesized derivatives 1-12. Hot plate method is generally used centrally umpired test grounded on thermal incentive with the contribution of spinal reflex action [39]. In this model, centrally interim analgesic agents constrain pain transmission at dorsal horn and persist the latency time of animals to jumping response [23][40]. Among derivatives 1-12, nine compounds were effective in antagonizing pain as depicted in table-2\&3 and graph $1 \& 2$.

Compound 1 displayed delay in latency time from 30-180 min. and maximum effect (66\%) was observed at 30 min. Compound $\mathbf{3}$ is most effective derivative of this series which antagonizes pain comparable to standard morphine. The thermally prompted jumping action of control mice revealed around 10-13sec from time interval of 0-180 mins. However the compound showed highly significant analgesia from 30-150 min (113-120\%) followed by gradual decline in latency time. Compound 4 showed analgesic response from 90-150 min. The maximum delay in latency time observed at $120 \mathrm{~min}$ (68\%). Compound 5 showed analgesia from 30-150 min. Compounds 10, 12 and 2 showed weak inhibition of pain. Compound 11 showed significant analgesic activity at $30 \mathrm{~min}$ (52\%) which was followed by decrease in latency time from 60-150 min and the effect was non-significant at $180 \mathrm{~min}$. Compound $\mathbf{6}$ displayed gradual increase in antinoceceptive activity from $30 \mathrm{~min}$ and the response is maximum at $120 \mathrm{~min}$ which was followed by gradual decrease from 150-180 min. The results was significant from $60-180 \mathrm{~min}$.

Compound 7 showed $88 \%$ analgesia at 30 min which was gradually decreased up to $29 \%$. Compound 8 showed onset of analgesic effect from $30 \mathrm{~min}$ and the result became highly significant (107\%) at $60 \mathrm{~min}$. Compound 9 exhibited significant inhibition of pain (76\%) at 30 min followed by rapid fall in activity.

Morphine sulphate at a dose of $10 \mathrm{mg} / \mathrm{kg}$ ip caused significant analgesia up to $30 \mathrm{~min}$ whereas gradual increase in latency time observed and the effect was highly significant from 60-180 min (61-176\%).

Regarding onset of action compounds $7, \mathbf{8}, \mathbf{9}, \mathbf{1}, \mathbf{3}$, and $\mathbf{5}$ displayed quick onset of action at 30 minutes while compounds $\mathbf{6}$ and 4 exhibited delayed onset of action at $60 \mathrm{~min}$ and 90 min respectively.

In case of duration of action compounds 3, 6, 1 and $\mathbf{5}$ have long duration (2-2.5 h), compounds $\mathbf{4}$ and $\mathbf{1 1}$ exhibited moderate (1-1.5 h) while compounds 7 and 9 have short duration of action ( $0.5 \mathrm{~h})$. The analgesic effect in terms of duration of action is given bellow.

\section{$3>6 / 1 / 5>8>4>11>7 / 9$}

Table-1: TFMP with Different Spacer and Linker. 


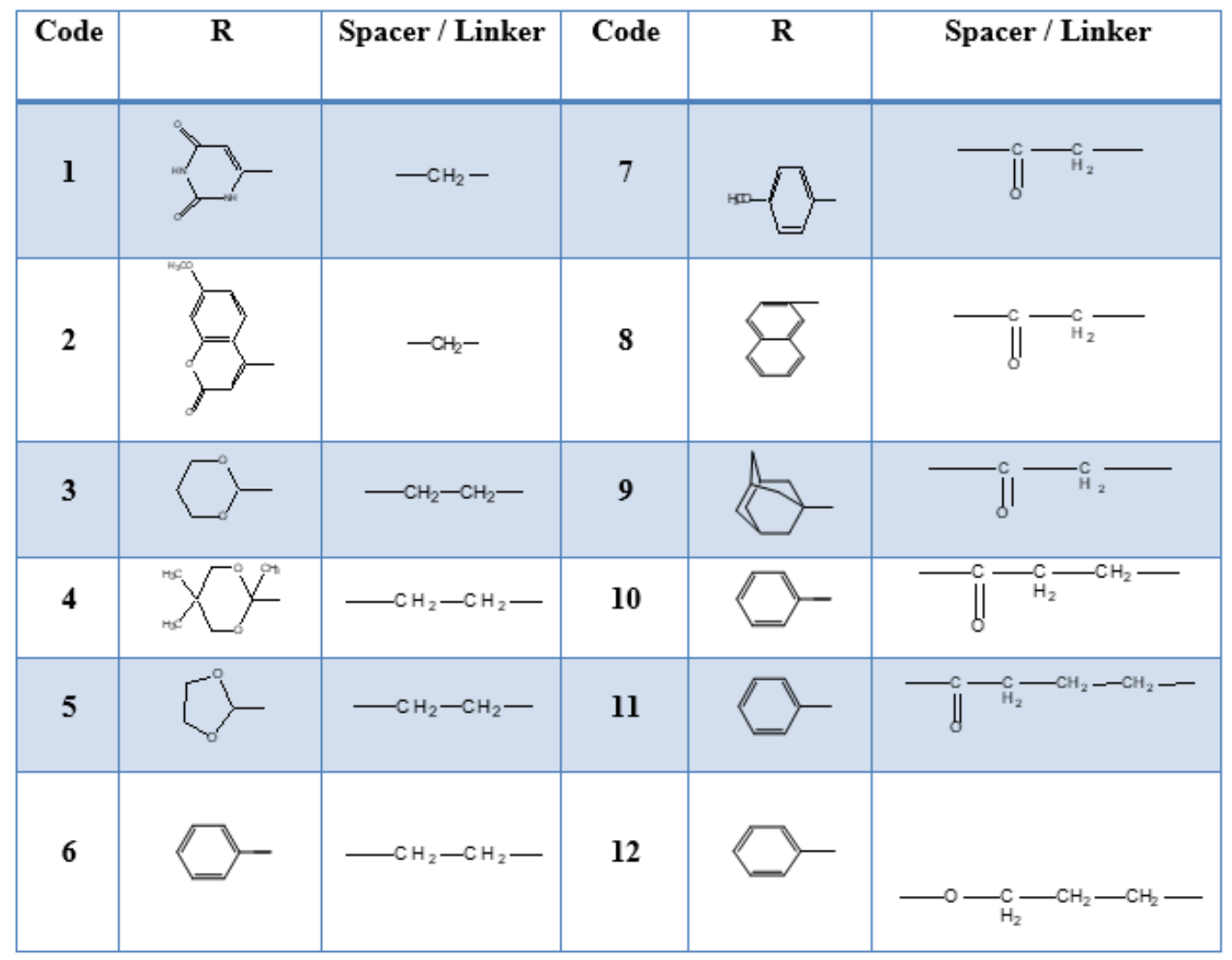

Structure-activity relationship (SAR) study specified that the activity of these 4-[4-chloro-3- (trifluoromethyl)-phenyl]-4piperidinol (TFMP) derivatives could be due to different acetophenone substituents. All the compounds possess three main regions as depicted in scheme- 2 where first region is same in all derivatives with variation is spacer and terminal ring system. All the synthesized derivatives are divided into two groups on the basis of SAR. First group $A\left(A_{1}-A_{3}\right)$ share the same substituted piperidine ring and spacer with the only difference in the terminal region. In second group $B$ piperidine and terminal ring are same with only difference in linking group. This only difference is responsible for varied results in analgesic activity.

In group $\mathrm{A}_{1}$, compounds $\mathbf{1}$ and $\mathbf{2}$ have methyl linkage between terminal ring and piperidine of parent molecule. Compound $\mathbf{1}$ showed significant analgesic activity due to the presence of uracil while substitution of coumarin moiety in compound $\mathbf{2}$, making the molecule completely inactive.

In group $\mathbf{A}_{\mathbf{2}}$, oxane (compound $\mathbf{3}$ ), 3, 5, 5 trimethyl oxane (compound $\mathbf{4}$ ), oxalane (compound 5) and phenyl group (compound 6) respectively are connected with piperidine ring via bridging of ethyl group. The presence of oxane in compound $\mathbf{3}$ and 3,5,5trimethyl oxane in compound $\mathbf{4}$ displayed interesting results as compounds $\mathbf{3}$ produced most pronounced and comparable to standard among all derivatives. Addition of three methyl group which is electron donating in nature in the same ring of compound $\mathbf{4}$ dropped the activity from highly significant to significant level. Another similar molecule compound $\mathbf{5}$ exhibited good analgesia but the presence of (5-membered) oxalane ring in place of oxane (6-membered) ring plays a crucial role in decreasing antinoceceptic effect. The presence of aromatic ring in compound $\mathbf{6}$, also produced comparable results as by compound $\mathbf{3}$ having oxane ring.

In group $\mathbf{A}_{\mathbf{3}}$, compounds7, $\mathbf{8}$, and $\mathbf{9}$ having substituted phenyl, naphthyl and adamantyl group respectively showed almost same level of analgesic activity. Significant anti-nociceptive effect exhibited by all these compounds 7, 8, and $\mathbf{9}$ suggesting the comparable ability of these three different ring systems to produce analgesic response.

In group B all the compounds $6,10,11$, and 12 have the same substituents but only difference is in connecting chain between the phenyl and piperidine ring. Among these only compound $\mathbf{6}$ produced highly significant effect, while the other provoked weaker analgesic response. SAR studies indicated the importance of ethyl linkage. Therefore it can be suggested that by replacing the $-\left(\mathrm{CH}_{2}\right)_{2}$ - with $-\mathrm{CO}-\left(\mathrm{CH}_{2}\right)_{2}-$ - $-\mathrm{CO}-\left(\mathrm{CH}_{2}\right)_{3}$ - and $-\mathrm{O}-\left(\mathrm{CH}_{2}\right)_{3}$ linkage analgesic activity was decreased. 


\section{Experimental}

\subsection{General Experimental Conditions}

All reagents used were obtained from Sigma Aldrich Company and organic solvents utilized were of analytical grade. Watt man's filter paper was used for filtration. TLC plates, Kieselgel 60 (GF-254) were used for the development of reaction and purification of compounds. The spots were envisaged under ultraviolet light at 254-366 nm on HP-UVIS Desaga (Heidelberg) using iodine vapors. The synthesized compounds were desiccated and stored in vacuum dry condition. Melting points of compounds were noted on Buchi 434 melting point apparatus and are uncorrected. Hitachi U-3200 spectrophotometer utilized to record ultraviolet (UV) spectra while methanol used as solvent. FT-IR spectra were investigated on a Jasco 302 Fourier transform infrared spectrophotometer by KBR disc method. Mass spectrometry were carried out on Varian Massen spectrometer MAT 311A spectrometer, Varian Massen spectrometer MAT 312, MAT 113 DMASPEC system. ${ }^{1} \mathrm{H}-\mathrm{NMR}$ and ${ }^{13} \mathrm{C}$ NMR spectral analysis was carried out at Bruker AM 300, spectrophotometer. Chemical shifts $(\delta)$ were recorded in parts per million (ppm) and coupling constants $J$ in Hertz.

\subsection{General procedure for Synthesis of TFMP Derivatives}

All derivatives have been synthesized by refluxing equimolar TFMP with a variety of acetophenone, dissolved separately in ethanol/acetone respectively in a RB flask. The RM was refluxed and stirred vigorously for 48 to 72 hours at $75^{\circ} \mathrm{C}$. The progress of reaction mixture was monitored by using TLC. Solid products were filtered and wash away numerous times with acetone. The resulting products were purified by the process of recrystallization using warm ethanol and diethyl ether/ dichloromethane. The pure product was dry in desiccators using anhydrous calcium sulphate.

\subsubsection{6-(2-\{4-[4-chloro-3-(trifluoromethyl)phenyl]-4-hydroxy-1- piperidinyl\}acetyl)-2,4(1H,3H)-pyrimidinedione hydrochloride (1)}

Yield: 79\%, White crystalline powder, mp: $285 \pm 2^{\circ} \mathrm{C}, \mathrm{C}_{24} \mathrm{H}_{22} \mathrm{NO}_{2} \mathrm{BrClF}_{3}$ (528.7889); Solubility (MeOH, EtOH, DMSO); UV ${ }_{\lambda \max }$ (MeOH) nm: 203, 206, 221, 268, 358; FT-IR $u_{\max }(\mathrm{KBr}) \mathrm{cm}^{-1}: 3381,2945,2773,1714,1506,1407,1324,1029,872,573$; El-MS m/z. 403.1 ( $\left.\mathrm{M}^{+}-\mathrm{HBr}\right), 385.1,278,260.1,207,180,166.1,126.1,82.9 ;$ HR-EIMS: 403. $0944\left(\mathrm{M}^{+}-\mathrm{HCl}_{1} \mathrm{C}_{17} \mathrm{H}_{17} \mathrm{~N}_{3} \mathrm{O}_{3} \mathrm{ClF}_{3}\right)$ (Calcd. 403.7832); UV Amax $(\mathrm{MeOH}) \mathrm{nm}: 203,206,221,268,358 ;{ }^{1} \mathrm{H}-\mathrm{NMR}(\mathrm{MeOD}, 300 \mathrm{MHz}) \delta \mathrm{H}: 7.92(\mathrm{~d}, 1 \mathrm{H}, J=2.1 \mathrm{~Hz}), 7.72(\mathrm{dd}, 1 \mathrm{H}$, $\left.J_{1}=2.1, J_{2}=8.4 \mathrm{~Hz}\right), 7.56(\mathrm{~d}, 1 \mathrm{H}, J=8.4 \mathrm{~Hz}), 5.64(\mathrm{~s}, 1 \mathrm{H}), 4.57(\mathrm{~s}, 1 \mathrm{H}, \mathrm{H}-14,3.33(\mathrm{~s}, 2 \mathrm{H}), 2.75(\mathrm{~d}, 2 \mathrm{H}, J=10.8 \mathrm{~Hz}), 2.63(\mathrm{~m}, 2 \mathrm{H})$, $2.18(\mathrm{~m}, 2 \mathrm{H}), 1.70$ (d, 2H, $J=12 \mathrm{~Hz}) ;{ }^{13} \mathrm{C}-\mathrm{NMR}(\mathrm{MeOD}, 100 \mathrm{MHz}$ ) $\delta \mathrm{C}(\mathrm{ppm}): 150.1,153.4,98.3,164.1,57.5,48.7,37.5,69.3$, $148.0,124.4,124.2,128.5,131.2,130.8,131.2$.

\subsubsection{4-(\{4-[4-chloro-3-(trifluoromethyl)phenyl]-4-hydroxy-1- piperidinyl\}methyl)-7-methoxy-2H-chromen-2-one hydrobromide (2)}

Yield: $88 \%$, White crystalline powder. $\mathrm{mp}: 281 \pm 2^{\circ} \mathrm{C}, \mathrm{C}_{24} \mathrm{H}_{26} \mathrm{NO}_{3} \mathrm{BrClF}_{3}$ (548.7770), Solubility (MeOH, EtOH, DMSO); UV ${ }_{\lambda \text { max }}$ $(\mathrm{MeOH}) \mathrm{nm}: 322,279,251,223,211$; FT-IR $U_{\max }(\mathrm{KBr}) \mathrm{cm}^{-1}: 3318,2899,2641,2568,1717,1612,1472,1390,1290,987,843,477$ El-MS m/z. 467.1 (M+-HBr), 448, 292, 190, 162, 147, 79.9; HR-EIMS: $467.1475\left(\mathrm{M}^{+}-\mathrm{HBr}, \mathrm{C}_{24} \mathrm{H}_{25} \mathrm{NO}_{3} \mathrm{ClF}_{3}\right)$ (Calcd. 467.8651); ${ }^{1} \mathrm{H}-$ NMR (MeOD, $300 \mathrm{MHz}) \delta \mathrm{H}: 7.93(\mathrm{~d}, 2 \mathrm{H}, J=10.2 \mathrm{~Hz}) 7.72(\mathrm{~d}, \mathrm{IH}, J=8.4 \mathrm{~Hz}), 7.56(\mathrm{~d}, 1 \mathrm{H}, J=8.4 \mathrm{~Hz}), 6.97(\mathrm{~m}, 2 \mathrm{H}), 6.40(\mathrm{~s}, 1 \mathrm{H})$, $3.88(\mathrm{~s}, 3 \mathrm{H}), 3.74(\mathrm{~s}, 2 \mathrm{H}), 2.85(\mathrm{~d}, 2 \mathrm{H}, \mathrm{J}=10.8 \mathrm{~Hz}), 2.70(\mathrm{t}, 2 \mathrm{H}, \mathrm{J}=10.5 \mathrm{~Hz}), 2.18(\mathrm{~m}, 2 \mathrm{H}), 1.74(\mathrm{~d}, 2 \mathrm{H}, J=12.3 \mathrm{~Hz}) ;{ }^{13} \mathrm{C}-\mathrm{NMR}$ (MeOD, $100 \mathrm{MHz}$ ) $\delta \mathrm{C}$ (ppm): 163.6, 156.9, 155.2, 150.5, 150.3, 132.6, 132.6, 131.2, 127.5, 126.3, 125.3, 125.1, 113.7, 113.5, $111.0,99.4,71.3,59.8,56.3,50.7,39.2$.

\subsubsection{4-[4-chloro-3-(trifluoromethyl)phenyl]-1-[2-(1, 3-dioxan-2-yl) ethyl]-4-piperidinol hydrobromide (3)}


Yield: $80 \%$, White crystalline powder, mp: $213 \pm 2^{\circ} \mathrm{C}, \mathrm{C}_{18} \mathrm{H}_{24} \mathrm{NO}_{3} \mathrm{ClBrF}_{3}(474.7400)$; Solubility (MeOH, EtOH, DMSO); UV $\mathrm{Vmax}_{\max }$ $(\mathrm{MeOH}) \mathrm{nm}: 223,266,344,387$; FT-IR $u_{\max }(\mathrm{KBr}) \mathrm{cm}^{-1}: 3741,3292,2931,2435,1831,1743,1647,1319,982,662,503$; El-MS m/z. 393 (M+-HBr), 374, 336, 307, 292, 274, 261,251, 201,101, 87; HR- ElMS: $393.1317\left(\mathrm{M}^{+}-\mathrm{HBr} \mathrm{C}_{18} \mathrm{H}_{23} \mathrm{NO}_{3} \mathrm{ClF}_{3}\right)$ (Calcd. 393.8281); ${ }^{1} \mathrm{H}-\mathrm{NMR}(\mathrm{MeOD}, 300 \mathrm{MHz}) \delta \mathrm{H}: 7.93(\mathrm{~s}, 1 \mathrm{H}), 7,74(\mathrm{~d}, 1 \mathrm{H}, J=8.4 \mathrm{~Hz}), 7.65(\mathrm{~d}, 1 \mathrm{H}, J=8.4 \mathrm{~Hz}), 7.30(\mathrm{~m}, 2 \mathrm{H}), 6.96(\mathrm{~m}$, $3 \mathrm{H}), 4.15(\mathrm{t}, 2 \mathrm{H}, J=5.7 \mathrm{~Hz}), 3.58(6 \mathrm{H}, \mathrm{m}), 2.37(\mathrm{~m}, 4 \mathrm{H}), 2.03(\mathrm{~d}, 2 \mathrm{H}, J=14.1 \mathrm{~Hz}) ;{ }^{13} \mathrm{C}-\mathrm{NMR}(\mathrm{MeOD}, 100 \mathrm{MHz}$ ) $\delta \mathrm{C}(\mathrm{ppm}): 148.4$, $132.0,131.4,130.5,128.3,124.7,124.1,68.4,66.0,52.0,49.1,35.5,30.3,25.2$.

\subsubsection{4-[4-chloro-3-(trifluoromethyl)phenyl]-1-[2-(2, 5, 5-trimethyl-1, 3- dioxan-2-yl) ethyl]-4-piperidinol hydrobromide (4)}

Yield: $75 \%$, White crystalline powder, $\mathrm{mp}: 244 \pm 2{ }^{\circ} \mathrm{C}, \mathrm{C}_{22} \mathrm{H}_{30} \mathrm{NO}_{3} \mathrm{ClBrF}_{3}$ (516.8197), Solubility (MeOH, EtOH, DMSO); UV $\mathbf{\lambda m a x}_{\max }$ (MeOH) nm: 268.2, 352, 389; IR $u_{\max }(\mathrm{KBr}) \mathrm{cm}^{-1}$ 3446, 3062, 2893, 2655, 435, 1747, 1580, 1480, 1387, 1129, 971, 905, 823, 658, 431; El-MS m/z. 435.2 (M+-HBr), 350.1, 292.1, 274.1, 235, 201, 129, 100.1; HR-EIMS: $435.1782\left(\mathrm{M}^{+}-\mathrm{HBr}, \mathrm{C}_{22} \mathrm{H}_{29} \mathrm{NO}_{3} \mathrm{CIF}_{3}\right)$ (Calcd. 435.9078); ${ }^{1} \mathrm{H}-\mathrm{NMR}(\mathrm{MeOD}, 300 \mathrm{MHz}) \delta \mathrm{H}: 7.93(\mathrm{~d}, 1 \mathrm{H}, J=1.6 \mathrm{~Hz}), 7.73(\mathrm{~d}, 1 \mathrm{H}, J=8.0 \mathrm{~Hz}), 7.64(\mathrm{~d}, 1 \mathrm{H}, J=8.8 \mathrm{~Hz}), 3.75$ (d, $2 \mathrm{H}, \mathrm{J}=11.6 \mathrm{~Hz}$ ), 3.55 (br. s, $2 \mathrm{H}), 3.476(\mathrm{~m}, 6 \mathrm{H}), 2.32(\mathrm{t}, 2 \mathrm{H}, J=11.6 \mathrm{~Hz}), 2.17(\mathrm{t}, 2 \mathrm{H}, \mathrm{J}=11.6 \mathrm{~Hz}), 2.17(\mathrm{t}, 2 \mathrm{H}, J=8.0 \mathrm{~Hz}), 2.00$ $(\mathrm{d}, 2 \mathrm{H}, J=14.0 \mathrm{~Hz}), 1.48(\mathrm{~s}, 3 \mathrm{H}), 10.11(\mathrm{~s}, 3 \mathrm{H}), 0.82(\mathrm{~s}, 3 \mathrm{H}) ;{ }^{13} \mathrm{C}-\mathrm{NMR}$ (MeOD, $100 \mathrm{MHz}$ ) $\delta \mathrm{C}$ (ppm): 148.3, 131.5, 131.5, 130.5, $129.0,128.3,124.0,97.3,69.2,68.3,48.4,35.4,35.4,33.0,22.4,21.9,20.3$.

\subsubsection{4-[4-chloro-3-(trifluoromethyl)phenyl]-1-[2-(1, 3-dioxolan-2-yl) ethyl]-4-piperidinol hydrobromide (5)}

Yield: $73 \%$, White crystalline powder, $\mathrm{mp}: 193 \pm 2^{\circ} \mathrm{C}, \mathrm{C}_{17} \mathrm{H}_{22} \mathrm{NO}_{3} \mathrm{ClBrF}_{3}(460.7134)$, Solubility (MeOH, EtOH, DMSO), UV $\mathrm{V}_{\mathrm{\lambda max}}$ $(\mathrm{MeOH}) \mathrm{nm}: 199.8,222,270,389 ; \mathrm{FT}-\mathrm{IR} u_{\max }(\mathrm{KBr}) \mathrm{cm}^{-1}:$ 3446, 2937, 2655, 2433, 1580, 1480, 1387, 1323, 1217, 1031,905, 658, 431, El-MS m/z. 379.2 (M+HBr), 279.1, 261.1, 207, 201, 167, 112 ,81.9, 153, 139, 124, 110, 83, 70, 55; HR-El-MS: 379.1156 (M+ . $\mathrm{HBr}, \mathrm{C}_{17} \mathrm{H}_{21} \mathrm{NO}_{3} \mathrm{ClF}_{3}$ ) (Calcd. 379.8015); ${ }^{1} \mathrm{H}-\mathrm{NMR}(\mathrm{MeOD}, 300 \mathrm{MHz}) \delta \mathrm{H}: 7.92(\mathrm{~s}, 1 \mathrm{H}), 7.73(\mathrm{~d}, 1 \mathrm{H}, \mathrm{J}=8.4 \mathrm{~Hz}), 7.64(\mathrm{~d}, 1 \mathrm{H}, J=8.4$ $\mathrm{Hz}), 5.00(\mathrm{t}, 1 \mathrm{H}, J=3.0 \mathrm{~Hz}), 4.03(\mathrm{~m}, 2 \mathrm{H}), 3.93(\mathrm{~m}, 2 \mathrm{H}), 3.55(\mathrm{~s}, 2 \mathrm{H}), 3.47(\mathrm{~m}, 4 \mathrm{H}), 2.32(\mathrm{~m}, 2 \mathrm{H}), 2.21(\mathrm{~m}, 2 \mathrm{H}), 2.00(\mathrm{~d}, 2 \mathrm{H}, J=14$ $\mathrm{Hz}) ;{ }^{13} \mathrm{C}-\mathrm{NMR}(\mathrm{MeOD}, 100 \mathrm{MHz}$ ) $\delta \mathrm{C}(\mathrm{ppm}): 148.1,132.8,132.0,131.1,126.2,125.6,125.3,102.6,69.1,66.2,54.2,50.2,35.6$, 29.3.

\subsubsection{4-[4-chloro-3-(trifluoromethyl)phenyl]-1-phenethyl-4-piperidinol hydrobromide (6)}

Yield: 86\%, White crystalline powder, mp: $191 \pm 2{ }^{\circ} \mathrm{C}, \mathrm{C}_{20} \mathrm{H}_{22} \mathrm{NOBrClF}_{3}$ (463.0525), Solubility (methanol, ethanol, DMSO); UV ${ }_{\lambda \max }$ $(\mathrm{MeOH}) \mathrm{nm}: 221.2,265.8,390$; El-MS m/z: 384 (M+-HBr), 364.1, 212, 194, 184, 158, 135, 116.4, 89, 63; FT-IR u max $(\mathrm{KBr}) \mathrm{cm}^{-1}$ : $3737,3342,2925,2564,1742,1645,1456,1319,1178,1033,725,546$; HR-FABMS: $384.123\left\{\left[\mathrm{M}+\mathrm{H}^{+}-\mathrm{HBr} \mathrm{C}_{20} \mathrm{H}_{22} \mathrm{NOCIF}_{3}\right\}\left(\mathrm{Calcd}_{\text {. }}\right.\right.$ 384.843); ${ }^{1} \mathrm{H}-\mathrm{NMR}(\mathrm{MeOD}, 300 \mathrm{MHz}) \delta \mathrm{H}: 7.78(\mathrm{~s}, 1 \mathrm{H}), 7.74(\mathrm{~m}, 2 \mathrm{H}), 7.24(\mathrm{~m}, 5 \mathrm{H}), 5.83(\mathrm{~s}, 1 \mathrm{H}), 3.54(\mathrm{~s}, 2 \mathrm{H}), 3.30(\mathrm{~s}, 2 \mathrm{H}), 3.03(\mathrm{t}, 4 \mathrm{H}$, $J=15.3 \mathrm{~Hz}), 2.26$ (t, 2H, $J=14.1 \mathrm{~Hz}), 1.86(\mathrm{~d}, 2 \mathrm{H}, J=14.1 \mathrm{~Hz}) ;{ }^{13} \mathrm{C}-\mathrm{NMR}(\mathrm{MeOD}, 100 \mathrm{MHz}) \delta$ (ppm): 138.3, 132.8, 131.9, 131.1, $129.9,129.8,126.2,125.2,122.6,64.6,59.4,50.1,36.8,32.1$.

\subsubsection{2-\{4-[4-chloro-3-(trifluoromethyl)phenyl]-4-hydroxy-1-piperidinyl\}-1- (4-methoxyphenyl)-1-ethanone hydrobromide (7)}

Yield: $80.5 \%$, White crystalline powder, $\mathrm{mp}: 275 \pm 3^{\circ} \mathrm{C}, \mathrm{C}_{21} \mathrm{H}_{22} \mathrm{NO}_{3} \mathrm{BrClF} 3$ (508.7562 a.m.u), Solubility (methanol, ethanol, DMSO), UV ${ }_{\lambda \max }(\mathrm{MeOH}) \mathrm{nm}: 203,278,390 \mathrm{FT}-\mathrm{IR} u_{\max }(\mathrm{KBr}) \mathrm{cm}^{-1}: 3742,3285,2929,2568,1683,1467,1319,1138,622 ;$ El-MS $\mathrm{m} / \mathrm{z}$. 428 (M+-HBr), 408, 292, 274, 207, 210, 135, 121.1, 56.1; HR- El-MS: $428.1230\left(\mathrm{M}^{+}-\mathrm{Br}_{,} \mathrm{C}_{21} \mathrm{H}_{22} \mathrm{NO}_{3} \mathrm{ClF}_{3}\right)$ (Calcd. 428); ${ }^{1} \mathrm{H}-$ NMR (MeOD, $300 \mathrm{MHz}) \delta \mathrm{H}:(\mathrm{ppm}): 8.01(\mathrm{~m}, 2 \mathrm{H}), 7.76(\mathrm{~d}, 1 \mathrm{H}, J=7.74 \mathrm{~Hz}), 7.64$ (d, 2H, J= 8.4 Hz), 7.09 (d, 2H, J=8.8 Hz), 3.90 $(\mathrm{s}, 3 \mathrm{H}),, 3.62(\mathrm{~d}, 4 \mathrm{H}, J=12.9 \mathrm{~Hz}), 2.48(\mathrm{~s}, 2 \mathrm{H}), 1.98$ (d, 2H, J=14 Hz); ${ }^{13} \mathrm{C}-\mathrm{NMR}$ (MeOD, $\left.100 \mathrm{MHz}\right) \delta \mathrm{C}(\mathrm{ppm}): 190.3,166.7,139.3$, $133.1,131.9,131.3,130.0,129.2,126.1,125.5,122.5,115.4,69.9,61.2,52.6,51.3,35.0,25.0$. 


\subsubsection{2-\{4-[4-chloro-3-(trifluoromethyl)phenyl]-4-hydroxy-1-piperidinyl\}-1- (2-naphthyl)-1-ethanone hydrobromide (8)}

Yield: $79 \%$, White crystalline powder, mp: $285 \pm 2^{\circ} \mathrm{C}, \mathrm{C}_{24} \mathrm{H}_{22} \mathrm{NO}_{2} \mathrm{BrClF}_{3}$ (528.7889); Solubility (MeOH, EtOH, DMSO); UV ${ }_{\lambda \max }$ (MeOH) nm: 199.8, 222, 250.2, 279, 338.2; FT-IRu $\max (K B r)$ cm$^{-1}: 3740,3442,2929,2657,1836,1685,1627,1399,1177,1137$, 1035, 816, 750, 473; El-MS m/z. 448 (M+HBr), 428, 291.9, 274, 257, 201, 178, 155, 127, 79.9; HR-EIMS: $448.1313\left(\mathrm{MH}^{+}-\mathrm{HBr}\right.$ ${ }^{,} \mathrm{C}_{24} \mathrm{H}_{22} \mathrm{~N}_{2} \mathrm{OClF}_{3}$ ) (Calcd. 448.8849); ${ }^{1} \mathrm{H}-\mathrm{NMR}(\mathrm{MeOD}, 300 \mathrm{MHz}) \delta \mathrm{H}: 8.69(\mathrm{~s}, 1 \mathrm{H}), 8.04(\mathrm{~m}, 4 \mathrm{H}), 7.80(\mathrm{~m}, 4 \mathrm{H}), 5.20(\mathrm{~s}, 2 \mathrm{H}), 3.70(\mathrm{~d}$, $4 \mathrm{H}, J=13.8 \mathrm{~Hz}), 2.59(\mathrm{~m}, 2 \mathrm{H}), 2.02(\mathrm{~d}, 2 \mathrm{H}, J=14.1 \mathrm{~Hz}) ;{ }^{13} \mathrm{C}-\mathrm{NMR}(\mathrm{MeOD}, 100 \mathrm{MHz},) \delta \mathrm{C}(\mathrm{ppm}): 196.2,150.0,134.6,133.9,132.9$, $132.8,132.6,131.6,131.5,130.3,128.9,126.3,69.9,61.2,51.0,35.6$.

\subsubsection{1-(1-adamantyl)-2-\{4-[4-chloro-3-(trifluoromethyl)phenyl]-4- hydroxy-1-piperidinyl-1-ethanone hydrobromide (9)}

Yield: 79\%, White crystalline powder, mp: $285 \pm 2^{\circ} \mathrm{C}, \mathrm{C}_{24} \mathrm{H}_{22} \mathrm{NO}_{2} \mathrm{BrClF}_{3}$ (528.7889), Solubility (MeOH, EtOH, DMSO); UV $\lambda_{\max }$ $(\mathrm{MeOH}) \mathrm{nm}: 271,385,389.6 \mathrm{FT}-\mathrm{IR} u_{\max }(\mathrm{KBr}) \mathrm{cm}^{-1}: 3315.6,2917,2856,1709,1582,449,1320,1212,1143,985,833$, EIMS m/z. 456 (M+-HBr), 436,292, 294, 274, 257, 232, 208, 201, 178, 135, 107, 93.1, 79.1,56; HR- FABMS: $456.1905\{\mathrm{M}+\mathrm{H}]^{+}-\mathrm{HBr}$ $\mathrm{C}_{24} \mathrm{H}_{30} \mathrm{NO}_{2} \mathrm{ClF}_{3}$ \} Calcd. 456.9484; ${ }^{1} \mathrm{H}-\mathrm{NMR}(\mathrm{MeOD}, 300 \mathrm{MHz}) \delta \mathrm{H}: 7.93(\mathrm{~d}, J=2.1 \mathrm{~Hz}), 7.72\left(\mathrm{dd}, 1 \mathrm{H}, J_{1}=2.1 \mathrm{~Hz}, \mathrm{~J}_{2}=8.4 \mathrm{~Hz}\right), 7.58$ $(\mathrm{m}, 1 \mathrm{H}), 3.54(\mathrm{~m}, 2 \mathrm{H}), 2.79(\mathrm{~d}, 2 \mathrm{H}, J=10.2 \mathrm{~Hz}), 2.61(\mathrm{~m}, 2 \mathrm{H}), 2.22(\mathrm{~m}, 2 \mathrm{H}), 2.03$ (br.s, 3H), $1.87(\mathrm{~m}, 7 \mathrm{H}), 1.71(\mathrm{~m}, 5 \mathrm{H}) ;{ }^{13} \mathrm{C}-\mathrm{NMR}$ (MeOD, 100 MHz) $\delta C$ (ppm): 207.5, 147.0, 130.4, 130.4, 129.2, 126.5, 124.7, 67.7, 59.8, 49.1, 44.9, 36.6, 35.7, 34.6, 27.0.

\subsubsection{3-\{4-[4-chloro-3-(trifluoromethyl)phenyl]-4-hydroxy-1- piperidinyl\}-1-phenyl-1-propanone hydrochloride (10)}

Yield: 83\%, White crystalline powder, mp: $220 \pm 2^{\circ} \mathrm{C}, \mathrm{C}_{21} \mathrm{H}_{22 n} \mathrm{NO}_{2} \mathrm{Cl}_{2} \mathrm{~F}_{3}$ (447.0979), Solubility (MeOH, EtOH, DMSO). 434; UV $\boldsymbol{\lambda}_{\max }$ (MeOH) nm: 201, 223, 243, 290, 302, 312, 316, 329. FT-IR U max $(K B r) \mathrm{cm}^{-1}:$ 3902, 3731, 3449, 2423, 3253, 2932, 2802, 2586, 2126, 1985, 1686, 1773, 1686, 1582, 1322, 1031, 668; El-MS m/z. 411 (M+-HBr), 306.1, 292, 279.1, 260.9, 251.0, 232.1, 207, 179.1, 144, 132.1, 105; HR-EIMS: $411.1185\left(\mathrm{M}^{+}-\mathrm{HCl}, \mathrm{C}_{21} \mathrm{H}_{22 n} \mathrm{NO}_{2} \mathrm{Cl}_{2} \mathrm{~F}_{3}\right.$ (Calcd. 411.8449); ${ }^{1} \mathrm{H}-\mathrm{NMR}$ (MeOD, $\left.300 \mathrm{MHz}\right) \delta \mathrm{H}: 10.59$ $(\mathrm{s}, 1 \mathrm{H}), 8.04(\mathrm{~s}, 1 \mathrm{H}), 8.01(\mathrm{~d}, 1 \mathrm{H}, J=1.2 \mathrm{~Hz}), 7.91(\mathrm{~s}, 1 \mathrm{H}), 7.75(\mathrm{~m}, 3 \mathrm{H}), 7.59(\mathrm{~m}, 2 \mathrm{H}), 5.84(\mathrm{~s}, 1 \mathrm{H}), 3.70(\mathrm{~s}, 2 \mathrm{H}), 3.51(\mathrm{~m}, 6 \mathrm{H}), 2.49$ (s, 1H) 1.86 (d, 2H, J= 13.8); ${ }^{13} \mathrm{C}-\mathrm{NMR}-(\mathrm{MeOD}, 100 \mathrm{MHz}) \delta \mathrm{C}$ (ppm): 197.7, 148.3, 136.3, 133.4, 132.3, 131.4, 130.2, 128.8, 128.4, $128.4,128.4,124.7,68.6,51.7,48.5,34.2,23.9$.

3.2.11 4-\{4-[4-chloro-3-(trifluoromethyl)phenyl]-4-hydroxy-1-piperidinyl\}-1-phenyl-1-butanone hydrochloride (11)

Yield: 73\%, White crystalline powder, mp: $223 \pm 2^{\circ} \mathrm{C}, \mathrm{C}_{22} \mathrm{H}_{24} \mathrm{NO}_{2} \mathrm{Cl}_{2} \mathrm{~F}_{3}$ (462.3321), Solubility (MeOH, EtOH, DMSO); UV ${ }_{\lambda \max }$ (MeOH) nm: 202, 242, 270, 302, 316, 319, 324, 336; IR $u_{\max }(\mathrm{KBr}) \mathrm{cm}^{-1}: 3286,2950,2894,2653,1680,1480,1319,1120,984$, 834, 652, 517; EIMS m/z. 425 (M+HBr), 405.9, 374.9, 304.9, 291.9, 273.9, 256.8, 200, 178.9, 105, 98; HR- EIMS: 425.136 (M+ $\mathrm{HCl}, \mathrm{C}_{22} \mathrm{H}_{23} \mathrm{NO}_{2} \mathrm{ClF}_{3}$ ) (Calcd. 425.8715); ${ }^{1} \mathrm{H}-\mathrm{NMR}(\mathrm{MeOD}, 300 \mathrm{MHz}) \delta \mathrm{H}: 8.03(\mathrm{~s}, 1 \mathrm{H}), 8.01(\mathrm{~d}, 1 \mathrm{H}, J=1.5 \mathrm{~Hz}), 7.92(\mathrm{~d}, 1 \mathrm{H}, J=2.1$ $\mathrm{Hz}), 7.72(\mathrm{dd}, J=1.8 \mathrm{~Hz}, J=8.4 \mathrm{~Hz}), 7.64(\mathrm{~m}, 2 \mathrm{H}), 7.53(\mathrm{~m}, 2 \mathrm{H}), 3.41(\mathrm{~d}, 2 \mathrm{H}, J=9.9 \mathrm{~Hz}), 3.23(\mathrm{t}, 4 \mathrm{H}, J=12.0 \mathrm{~Hz}), 3.11(\mathrm{t}, 2 \mathrm{H}, J=$ $7.8 \mathrm{~Hz}), 2.29$ (m, 4H), 1.09 (m, 2H); ${ }^{13} \mathrm{C}-\mathrm{NMR}$ (MeOD, $100 \mathrm{MHz}$ ) $\delta \mathrm{C}(\mathrm{ppm}): 198.6,148.0,136.4,133.2,131.6,131.2,130.4,129.1$, $128.7,127.8,124.0,124.0,68.1,55.3,48.2,35.2,34.7$.

3.2.12 4-[4-chloro-3-(trifluoromethyl)phenyl]-1-(3-phenoxypropyl)-4-piperidinol hydrobromide (12)

Yield: $80 \%$, White crystalline powder, $\mathrm{mp}: 179 \pm 2^{\circ} \mathrm{C}, \mathrm{C}_{21} \mathrm{H}_{24} \mathrm{NO}_{2} \mathrm{ClBrF}_{3}$ (494.7727); Solubility (MeOH, EtOH, DMSO); $\mathrm{UV}_{\lambda \max }$ $(\mathrm{MeOH}) \mathrm{nm}: 201,221,270,277,302,324$; FT-IR U $_{\max }(\mathrm{KBr}) \mathrm{cm}^{-1}: 3447,3422,3284,2924,2649,2432,1782,1598,1321,1125$, 766, 427; El-MS m/z. 413.2 (M+-HBr), 306, 394.2, 320.1, 292.1, 274.1, 276.1, 261.1, 207, 201.1, 179, 107, 94; HR- EIMS: $413.1380\left(\mathrm{M}^{+}-\mathrm{HBr}, \mathrm{C}_{21} \mathrm{H}_{23} \mathrm{NO}_{2} \mathrm{ClF}_{3}\right.$ ) (Calcd. 413.1369); ${ }^{1} \mathrm{H}-\mathrm{NMR}(\mathrm{MeOD}, 300 \mathrm{MHz}) \delta \mathrm{H}: 7.93(\mathrm{~s}, 1 \mathrm{H}), 7.74(\mathrm{~d}, 1 \mathrm{H}, J=8.4 \mathrm{~Hz}), 7.65$ 
$(\mathrm{d}, 1 \mathrm{H}, J=8.4 \mathrm{~Hz}), 7.30(\mathrm{~m}, 2 \mathrm{H}), 6.96(\mathrm{~m}, 3 \mathrm{H}), 4.15(\mathrm{t}, 2 \mathrm{H}, J=5.7 \mathrm{~Hz}), 3.58(6 \mathrm{H}, \mathrm{m}), 2.37(\mathrm{~m}, 4 \mathrm{H}), 2.03(\mathrm{~d}, 2 \mathrm{H}, J=14.1 \mathrm{~Hz}) ;{ }^{13} \mathrm{C}-$ NMR (MeOD, $100 \mathrm{MHz}$ ) $\delta \mathrm{C}$ (ppm): 159.9, 148.0, 132.8, 132.1, 131.1, 148.0, 126.2, 125.4, 122.6, 122.2, 115.5, 69.2, 65.8, 50.2, 49.8, 36.5, 25.5 .

\subsection{Biological Activity Evaluation}

\subsubsection{Animals}

The animal used in the study were facilitated by International Center for Chemical and Biological Sciences, H.E.J. Research Institute of Chemistry, University of Karachi, Pakistan. The ethical strategies for the treatment of laboratory animals were followed as suggested by Association for Assessment and Accreditation of laboratory Animal Care International (AAALAC) through approval of animal use committee. The NMRI mice of both sexes (23-29 g) were retained at a controlled temperature $\left(22 \pm 2{ }^{\circ} \mathrm{C}\right)$ and clamminess $(50 \pm 10 \%)$ with $12 \mathrm{~h}$ light and dark cycle and nourished with standard diet and water

\subsubsection{Sample Preparation}

Derivatives of 4-[4-chloro-3- (trifluoromethyl)-phenyl]-4-piperidinol(TFMP) were analyzed orally for analgesic influence at the operative dose of $50 \mathrm{mg} / \mathrm{Kg}$ (as $25 \mathrm{mg} / \mathrm{Kg}$ was found ineffective and $100 \mathrm{mg} / \mathrm{Kg}$ weight was observed lethal). 0.5\% gum tragacanth and morphine sulphate $(10 \mathrm{mg} / \mathrm{Kg})$ ip were used as drug vehicle and standard respectively.

\subsubsection{Hot Plate Method}

Eddy's hot plate methodology was utilized to investigate the analgesic activity of the synthesized derivatives 1-12 [24]. Mice ( $n=6 /$ dose) were orally pretreated with each vehicle (0.5\% gum tragacanth suspension), synthesized drugs $(50 \mathrm{mg} / \mathrm{Kg}) \mathrm{and}$ morphine sulphate $\left(10 \mathrm{mg} / \mathrm{kg}\right.$, ). Each mice was placed on hot plate (Ugo Basile, model-DS $37,25 \times 25 \mathrm{~cm}$, Italy) at $50 \pm 1^{\circ} \mathrm{C}$ temperature, just after giving the dose. The responding time of the animal to the pain stimulus by jumping or flicking or licking of hind paw licking is the latency time which was recorded in seconds. The detach time for the reaction was 30 sec to evade tissue damages [25]. Analgesic activity was monitored at $0,30,60,90,120,150$, and 180 minutes and outcomes were concise in the form of latency time. The average latency time displayed by the control animals (base line latency) and treated group (drug latency) were matched and stated as percent pain protection via following formula:

\subsubsection{Statistical Analysis}

One way analysis of variance (ANOVA) analgesic activity was expressed as latency \pm SEM in second. The alterations between the means were tested utilizing post hoc LSD and values of $p<0.05$ were statistically determined as significant. Statistical analysis was done on SPSS for windows version 12.

\subsection{Conclusion}

We have synthesized a series of 4-[4-chloro-3- (trifluoromethyl)-phenyl]-4-piperidinol (TFMP) derivatives. The reactions of different derivatives of 4-[4-chloro-3- (trifluoromethyl)-phenyl]-4-piperidinol (TFMP) took place with a variety of acetophenones in extraordinary yields, because of electronic effects. The synthesized compounds were characterized by using different techniques such as FT-IR, UV, ${ }^{1} \mathrm{H}-\mathrm{NMR},{ }^{13} \mathrm{C}-\mathrm{NMR}$ and mass spectrometry.

In vivo biological potential of TFMP derivatives 1-12 was evaluated by using Eddy's hot plate methodology. All synthesized compounds except 10, 12 and 2 displayed considerable inhibition of pain in the mouse hot plate model following administration by the oral route. Compounds $3,5,6$, and $\mathbf{8}$ induce potent analgesic effect. As the hot plate technique was employed thereby, imply that analgesic outcome is neurogenic possibly via participation of opioid receptors at spinal/supra spinal level. Overall, the preclinical data for this class of substituted piperidines signify them as novel class of effective analgesics, for potential therapeutic activity, which possibly will be devoid of the antagonistic side effects related with existing opioid analgesic treatments. Due to their encouraging results, they can be selected for relieving pain. On the other hand, contribution of particular receptor(s) in this analgesic potential needs to be determined. 
Table-2: Latency time (Percent Pain protection) of TFM derivatives and morphine on centrally mediated pain using hot plate test in mice.

\begin{tabular}{|c|c|c|c|c|c|c|c|c|}
\hline \multirow[t]{2}{*}{ Treatment } & \multirow[t]{2}{*}{$\begin{array}{l}\text { Dose } \\
\mathrm{mg} / \mathrm{kg}\end{array}$} & \multicolumn{7}{|c|}{$\begin{array}{l}\text { Latency time in sec } \\
\text { (Percent analgesia) }\end{array}$} \\
\hline & & Omin & $30 \mathrm{~min}$ & $60 \mathrm{~min}$ & $90 \mathrm{~min}$ & $120 \mathrm{~min}$ & $150 \mathrm{~min}$ & $180 \mathrm{~min}$ \\
\hline \multirow[t]{2}{*}{1} & \multirow[t]{12}{*}{$\begin{array}{l}50 \\
\mathrm{mg} / \mathrm{Kg}(\mathrm{po})\end{array}$} & $\begin{array}{l}11.38 \\
\pm .32\end{array}$ & $\underset{\star \star \star \star}{22.61 \pm .40}$ & $\begin{array}{l}19.85 \\
\pm .64 \star \star \star\end{array}$ & $\begin{array}{l}19.45 \\
\pm .39 \star \star \star\end{array}$ & $\begin{array}{l}18.73 \\
\pm .32^{\star \star \star}\end{array}$ & $\begin{array}{l}18.56 \\
+.26^{\star \star \star}\end{array}$ & $\begin{array}{l}18.46 \\
+.37 \star \star \star\end{array}$ \\
\hline & & $(7.8)$ & $(66)$ & $(43.7)$ & $(52.7)$ & $(49.7)$ & $(50.8)$ & $(46.6)$ \\
\hline \multirow[t]{2}{*}{2} & & $\begin{array}{l}11.22 \\
\pm .36\end{array}$ & $\begin{array}{l}15.24 \pm \\
1.20\end{array}$ & $\begin{array}{l}16.06 \\
+.30^{\star *}\end{array}$ & $\begin{array}{l}17.62 \\
\pm .98^{\star \star \star}\end{array}$ & $\begin{array}{l}14.24 \pm \\
1.01\end{array}$ & $\begin{array}{l}12.98 \\
\pm .44\end{array}$ & $\begin{array}{l}13.78 \\
\pm .65\end{array}$ \\
\hline & & $(6.1)$ & $(12.9)$ & $(16.2)$ & $(38.4)$ & $(13.8)$ & $(5.5)$ & $(9.4)$ \\
\hline \multirow[t]{2}{*}{3} & & $\begin{array}{l}11.35 \\
+.28\end{array}$ & $\begin{array}{l}28.77+ \\
2.63^{\star \star \star}\end{array}$ & $\begin{array}{l}33.25+ \\
2.26^{\star \star \star}\end{array}$ & $\begin{array}{l}36.71+ \\
3.08^{\star \star \star}\end{array}$ & $\begin{array}{l}27.54+ \\
1.92^{\star \star \star}\end{array}$ & $\begin{array}{l}19.64 \\
+.84^{\star \star \star}\end{array}$ & $\begin{array}{l}19.62 \\
+.98^{\star \star \star}\end{array}$ \\
\hline & & $(7.5$ & $(113.2$ & $(140.7$ & $(188$ & $(120$ & (59.6 & $(55.8$ \\
\hline \multirow[t]{2}{*}{4} & & $\begin{array}{l}10.72 \\
\pm .32\end{array}$ & $\begin{array}{l}16.38 \\
\pm .46^{\star \star \star}\end{array}$ & $\begin{array}{l}17.54 \\
\pm .51 * \star \star\end{array}$ & $\begin{array}{l}18.46 \\
\pm .60 \star \star \star \star\end{array}$ & $\begin{array}{l}21.10 \\
\pm .29 * \star \star\end{array}$ & $\begin{array}{l}18.00 \\
\pm .29 \star \star \star\end{array}$ & $\begin{array}{l}16.31 \pm \\
1.56 \star \star \star\end{array}$ \\
\hline & & $(1.6)$ & $(21.4)$ & $(27.0)$ & $(45.0)$ & $(68.6)$ & $(46.3)$ & $(22.8)$ \\
\hline \multirow[t]{2}{*}{5} & & $\begin{array}{l}11.30 \\
\pm .26\end{array}$ & $\begin{array}{l}32.08 \pm \\
2.37^{* \star \star}\end{array}$ & $\begin{array}{l}23.96 \\
+.47^{\star \star \star}\end{array}$ & $\begin{array}{l}23.85 \\
+.6^{\star \star \star 4}\end{array}$ & $\begin{array}{l}23.88 \pm \\
1.36 * \star \star\end{array}$ & $\begin{array}{l}19.64 \\
\pm .84^{\star \star \star}\end{array}$ & $\begin{array}{l}14.37 \\
\pm .55^{\star \star}\end{array}$ \\
\hline & & (7.1) & (137.8) & $(73.4)$ & (87.3) & $(90.8)$ & $(59.6)$ & $(14.1)$ \\
\hline \multirow[t]{2}{*}{6} & & $\begin{array}{l}11.21 \\
\pm .32\end{array}$ & $\begin{array}{l}16.02 \\
\pm .62^{\star \star \star}\end{array}$ & $\begin{array}{l}23.48 \\
\pm .57 \star \star \star\end{array}$ & $\begin{array}{l}24.14 \\
\pm .44^{\star \star \star}\end{array}$ & $\begin{array}{l}32.86 \\
\pm .79 \star \star \star\end{array}$ & $\begin{array}{l}22.84 \\
\pm .67 \star \star \star\end{array}$ & $\begin{array}{l}19.84 \\
+.48^{\star \star \star}\end{array}$ \\
\hline & & $(6.3)$ & (18.75) & (70) & $(89.6)$ & (162.6) & $(85.6)$ & $(57.5)$ \\
\hline \multirow{2}{*}{$\begin{array}{l}\text { Morphine } \\
\text { Sulphate }\end{array}$} & $\begin{array}{l}10 \mathrm{mg} / \mathrm{Kg} \\
\text { (ip) }\end{array}$ & $\begin{array}{l}11.20 \pm \\
041\end{array}$ & $\ln _{\star \star \star}^{21.76 \pm .48}$ & $\underset{\star \star \star \star}{26.21 \pm .28}$ & ${ }_{\star \star \star \star}^{31.28} \pm .66$ & $\begin{array}{l}34.14 \pm \\
1.03^{\star \star \star}\end{array}$ & $\begin{array}{l}33.98 \\
+.71 \star \star \star\end{array}$ & $\begin{array}{l}31.98 \\
\pm .45^{\star \star \star}\end{array}$ \\
\hline & & $(6.1)$ & (61.3) & (89.7) & $(145.7)$ & (172.9) & $(176.2)$ & (154.0) \\
\hline \multirow[t]{2}{*}{ Control } & $\begin{array}{l}\text { Gum } \\
\text { tragacanth }\end{array}$ & $\begin{array}{l}10.55 \\
\pm .20\end{array}$ & $13.49 \pm .18$ & $13.81 \pm .29$ & $12.73 \pm .27$ & $12.51 \pm .25$ & $\begin{array}{l}12.30 \\
\pm .23\end{array}$ & $\begin{array}{l}12.59 \\
\pm .24\end{array}$ \\
\hline & $(0.5 \%)$ & & & & & & & \\
\hline
\end{tabular}

Asterisks indicate * $(p<0.05), * *(p<0.001)$ and *** $(p<0.005)$; Latency time $(\mathrm{sec})$ of control $(n=90)$ and treated groups $(n=$ $15)$, which are mean \pm SEM of animals in three independent experiment. Values represented in parenthesis are percent pain protection from centrally mediated pain.

Table-3: Latency time (Percent Pain Protection) of TFM derivatives and morphine on centrally mediated pain using hot plate test in mice. 


\begin{tabular}{|c|c|c|c|c|c|c|c|c|}
\hline \multirow[t]{2}{*}{ Treatments } & \multirow[t]{2}{*}{$\begin{array}{l}\text { Dose } \\
\mathrm{mg} / \mathrm{kg}\end{array}$} & \multicolumn{7}{|c|}{$\begin{array}{l}\text { Latency time (sec) } \\
\text { (Percent analgesia) }\end{array}$} \\
\hline & & Omin & $30 \mathrm{~min}$ & $60 \mathrm{~min}$ & $90 \mathrm{~min}$ & 120min & $150 \mathrm{~min}$ & $180 \mathrm{~min}$ \\
\hline 7 & $50 \mathrm{mg} / \mathrm{Kg}(\mathrm{po})$ & $\begin{array}{l}10.49 \\
\pm .28 \\
(-0.5)\end{array}$ & $\begin{array}{l}25.40 \\
\pm .29 \star \star \star \\
(88.2)\end{array}$ & $\begin{array}{l}16.7 \pm .89 \star \star \star \\
(20.9)\end{array}$ & $\begin{array}{l}16.31 \\
\pm .87^{\star \star \star} \\
(28)\end{array}$ & $\begin{array}{l}16.58 \\
\pm .87 \star \star \star \\
(32.5)\end{array}$ & $\begin{array}{l}16.32 \\
+.81^{\star \star \star} \\
(32.6)\end{array}$ & $\begin{array}{l}16.28 \\
+.61^{\star \star \star} \\
(29.3)\end{array}$ \\
\hline 8 & & $\begin{array}{l}10.04 \\
\pm .32 \\
(-4.8)\end{array}$ & $\begin{array}{l}19.70 \\
\pm .1 .06 \star \star \star \\
(46.3)\end{array}$ & $\begin{array}{l}28.67 \pm \\
1.28 * \star \star \\
(107.6)\end{array}$ & $\begin{array}{l}18.26+ \\
1.2^{\star \star \star} \\
(43.4)\end{array}$ & $\begin{array}{l}18.08 \pm \\
179 \star \star \star \\
(44.5)\end{array}$ & $\begin{array}{l}17.33 \\
\pm .1 .37^{\star \star \star} \\
(40.8)\end{array}$ & $\begin{array}{l}16.31 \\
\pm .22^{\star \star \star} \\
(29.5)\end{array}$ \\
\hline 9 & & $\begin{array}{l}11.09 \\
\pm .35 \\
(5.1)\end{array}$ & $\begin{array}{l}23.76 \\
\pm .72^{\star \star \star} \\
(76.13)\end{array}$ & $\begin{array}{l}16.50 \pm .33^{\star \star \star} \\
(19.4)\end{array}$ & $\begin{array}{l}16.04 \\
+.28 * \star \star \\
(26.0)\end{array}$ & $\begin{array}{l}16.00 \\
\pm .30 * \star \star \\
(27.8)\end{array}$ & $\begin{array}{l}15.55 \\
\pm .38^{\star \star \star} \\
(26.4)\end{array}$ & $\begin{array}{l}14.52 \\
\pm .49 \star \star \star \\
(15.3)\end{array}$ \\
\hline 10 & & $\begin{array}{l}11.33 \\
\pm .33 \\
(7.3)\end{array}$ & $\begin{array}{l}15.00 \\
+.86^{\star} \\
(11.1)\end{array}$ & $\begin{array}{l}18.60+.82^{\star \star \star} \\
(34.6)\end{array}$ & $\begin{array}{l}16.34 \\
+.80 \star \star \star \\
(28.3)\end{array}$ & $\begin{array}{l}12.60 \\
+.46 \\
(0.7)\end{array}$ & $\begin{array}{l}12.38 \\
+.32 \\
(0.6)\end{array}$ & $\begin{array}{l}12.76 \\
+.45 \\
(1.35)\end{array}$ \\
\hline 11 & & $\begin{array}{l}11.10 \\
\pm .38 \\
(5.5)\end{array}$ & $\begin{array}{l}20.54 \pm \\
1.02^{\star \star \star} \\
(52.2)\end{array}$ & $\begin{array}{l}19.36 \pm .42^{\star \star \star} \\
(40.1)\end{array}$ & $\begin{array}{l}15.95 \\
+.26^{\star \star \star} \\
(25.2)\end{array}$ & $\begin{array}{l}15.56 \\
+.25^{\star \star \star} \\
(24.3)\end{array}$ & $\begin{array}{l}15.41 \\
+.46 * \star \star \\
(25.2)\end{array}$ & $\begin{array}{l}12.64 \\
+.25 \\
(0.3)\end{array}$ \\
\hline 12 & & $\begin{array}{l}10.01 \\
\pm .26 \\
(-5.1)\end{array}$ & $\begin{array}{l}15.45 \\
\pm .83^{\star \star \star} \\
(14.5)\end{array}$ & $\begin{array}{l}16.25 \pm .34 \text { ** } \\
(17.6)\end{array}$ & $\begin{array}{l}15.44 \\
+.44^{\star \star \star} \\
(21.2)\end{array}$ & $\begin{array}{l}15.26 \\
\pm .31^{\star \star \star} \\
(21.5)\end{array}$ & $\begin{array}{l}14.89 \\
+.64^{\star \star \star} \\
(21.0)\end{array}$ & $\begin{array}{l}12.83 \\
\pm .38 \\
(1.9)\end{array}$ \\
\hline $\begin{array}{l}\text { Morphine } \\
\text { Sulphate }\end{array}$ & $10 \mathrm{mg} / \mathrm{Kg}$ (ip) & $\begin{array}{l}11.20 \\
\pm 041 \\
(6.1)\end{array}$ & 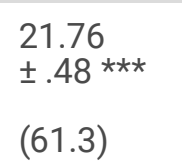 & $\begin{array}{l}\underset{\star \star \star}{26.21 \pm .28} \\
(89.7)\end{array}$ & $\begin{array}{l}31.28 \pm .66 \\
\star * \star \\
(145.7)\end{array}$ & $\begin{array}{l}34.14 \pm \\
1.03^{\star \star \star} \\
(172.9)\end{array}$ & $\begin{array}{l}33.98 \\
+.71 * \star \star \\
(176.2)\end{array}$ & $\begin{array}{l}31.98 \\
\pm .45^{\star \star \star} \\
(154.0)\end{array}$ \\
\hline Control & $\begin{array}{l}\text { Gum } \\
\text { tragacanth( } 0.5 \%)\end{array}$ & $\begin{array}{l}10.55 \\
\pm .20\end{array}$ & $\begin{array}{l}13.49 \\
\pm .18\end{array}$ & $13.81 \pm .29$ & $12.73 \pm .27$ & $\begin{array}{l}12.51 \\
\pm .25\end{array}$ & $\begin{array}{l}12.30 \\
\pm .23\end{array}$ & $\begin{array}{l}12.59 \\
\pm .24\end{array}$ \\
\hline
\end{tabular}

Asterisks indicate * $(p<0.05), * *(p<0.001)$ and $* * *(p<0.005)$; Latency time (sec.) of control $(n=90)$ and treated groups ( $n=$ $15)$, which are mean \pm SEM of animals in three independent experiment; Values represented in parenthesis are percent pain protection from centrally mediated pain.

\section{References}

1. Apkarian AV, Baliki MN, Geha PY: Towards a theory of chronic pain. Progress in neurobiology 2009, 87(2):81-97.

2. Le Bars D, Gozariu M, Cadden SW: Animal models of nociception. Pharmacological reviews 2001, 53(4):597-652.

3. Coutaux A, Adam F, Willer J-C, Le Bars D: Hyperalgesia and allodynia: peripheral mechanisms. Joint Bone Spine 2005, 72(5):359-371.

4. Chan HCS, McCarthy D, Li J, Palczewski K, Yuan S: Designing Safer Analgesics via $\mu$-Opioid Receptor Pathways. Trends in Pharmacological Sciences 2017, 38(11):1016-1037.

5. Dhargawe N, Mahakalkar S, Mohod B, Raj JP: Evaluation of analgesic, anti-inflammatory, and antipyretic activity of piperine: An experimental study. Pharmacognosy Research 2020, 12(2):176.

6. Sokeng SD, Talla E, Sakava P, Fokam Tagne MA, Henoumont C, Sophie L, Mbafor JT, Tchuenguem Fohouo F-N: Antiinflammatory and analgesic effect of arachic acid ethyl ester isolated from propolis. BioMed Research International 2020, 2020.

7. Goehring RR, Chen Z, Victory S, Kyle D: Benzimidazolone compounds. In.: Google Patents; 2005. 
8. Xiong J, Jin J, Gao L, Hao C, Liu X, Liu B-F, Chen Y, Zhang G: Piperidine propionamide as a scaffold for potent sigma-1 receptor antagonists and mu opioid receptor agonists for treating neuropathic pain. European journal of medicinal chemistry 2020, 191:112144.

9. Zhuang T, Xiong J, Hao S, Du W, Liu Z, Liu B, Zhang G, Chen Y: Bifunctional $\mu$ opioid and $\sigma 1$ receptor ligands as novel analgesics with reduced side effects. European Journal of Medicinal Chemistry 2021:113658.

10. Janssen PA, Niemegeers CJ, Dony JG: The inhibitory effect of fentanyl and other morphine-like analgesics on the warm water induced tail withdrawl reflex in rats. Arzneimittelforschung 1963, 13:502-507.

11. Thore SN, Gupta SV, Baheti KG: Synthesis and pharmacological evaluation of 5-methyl-2-phenylthiazole-4-substituted heteroazoles as a potential anti-inflammatory and analgesic agents. Journal of Saudi Chemical Society 2016, 20:S46-S52.

12. Vardanyan R: Chapter 7 - Piperidine-Based Nonfused Biheterocycles With C-N and C-C Coupling. In: Piperidine-Based Drug Discovery. Edited by Vardanyan R: Elsevier; 2017: 241-267.

13. Vardanyan R: Chapter 2 - 1-Substituted Piperidines. In: Piperidine-Based Drug Discovery. Edited by Vardanyan R: Elsevier; 2017: 83-101.

14. Bartolozzi A, Cirillo PF, Berry AK, Hickey ER, Thomson DS, Wu L, Zindell R, Albrecht C, Ceci A, Gemkow MJ: Selective CB2 receptor agonists. Part 3: The optimization of a piperidine-based series that demonstrated efficacy in an in vivo neuropathic pain model. Bioorganic \& medicinal chemistry letters 2015, 25(3):587-592.

15. Erol DD, Demirdamar R: Analgesic and antiinflammatory activity screening of 6-acyl-3-piperidinomethyl-2(3H)benzoxazolone derivatives. Farmaco (Societa chimica italiana : 1989) 1994, 49(10):663-666.

16. Miguel OG, Santos AR, Calixto JB, Delle Monache F, Yunes RA: Antinociceptive activity of the natural piperidine alkaloid hydrochlorides from Syphocampylus verticellatus. Zeitschrift fur Naturforschung C, Journal of biosciences 2002, 57(12):81-84.

17. Palin R, Clark JK, Evans L, Houghton AK, Jones PS, Prosser A, Wishart G, Yoshiizumi K: Structure-activity relationships and CoMFA of N-3 substituted phenoxypropyl piperidine benzimidazol-2-one analogues as NOP receptor agonists with analgesic properties. Bioorg Med Chem 2008, 16(6):2829-2851.

18. Zhang S, Su R, Zhang C, Liu X, Li J, Zheng J: C101, a novel 4-amino-piperidine derivative selectively blocks N-type calcium channels. European journal of pharmacology 2008, 587(1-3):42-47.

19. Chaudhari SS, Kadam AB, Khairatkar-Joshi N, Mukhopadhyay I, Karnik PV, Raghuram A, Rao SS, Vaiyapuri TS, Wale DP, Bhosale VM et al: Synthesis and pharmacological evaluation of novel N-aryl-3,4-dihydro-1'H-spiro[chromene-2,4'piperidine]-1'-carboxamides as TRPM8 antagonists. Bioorg Med Chem 2013, 21(21):6542-6553.

20. Lee JH, Seo SH, Lim EJ, Cho NC, Nam G, Kang SB, Pae AN, Jeong N, Keum G: Synthesis and biological evaluation of 1(isoxazol-5-ylmethylaminoethyl)-4-phenyl tetrahydropyridine and piperidine derivatives as potent T-type calcium channel blockers with antinociceptive effect in a neuropathic pain model. Eur J Med Chem 2014, 74:246-257.

21. Hayashi S, Nakata E, Morita A, Mizuno K, Yamamura K, Kato A, Ohashi K: Discovery of \{1-[4-(2-\{hexahydropyrrolo[3,4c]pyrrol-2(1H)-yl-1H-benzimidazol-1-yl)piperidin-1-yl]cyclooctyl\}methanol, systemically potent novel non-peptide agonist of nociceptin/orphanin FQ receptor as analgesic for the treatment of neuropathic pain: design, synthesis, and structureactivity relationships. Bioorg Med Chem 2010, 18(21):7675-7699.

22. Attalah KM, Abdalla AN, Aslam A, Ahmed M, Abourehab MA, ElSawy NA, Gouda AM: Ethyl benzoate bearing pyrrolizine/indolizine moieties: Design, synthesis and biological evaluation of anti-inflammatory and cytotoxic activities. Bioorganic chemistry 2020, 94:103371.

23. Abd El-Karim SS, Mohamed HS, Abdelhameed MF, Amr AE-GE, Almehizia AA, Nossier ES: Design, synthesis and molecular docking of new pyrazole-thiazolidinones as potent anti-inflammatory and analgesic agents with TNF- $a$ inhibitory activity. Bioorganic Chemistry 2021, 111:104827.

24. Eddy NB, Leimbach D: Synthetic analgesics. Il. Dithienylbutenyl- and dithienylbutylamines. The Journal of pharmacology and experimental therapeutics 1953, 107(3):385-393. 
25. Mekonnen T, Urga K, Engidawork E: Evaluation of the diuretic and analgesic activities of the rhizomes of Rumex abyssinicus Jacq in mice. Journal of ethnopharmacology 2010, 127(2):433-439.

\section{Figures}

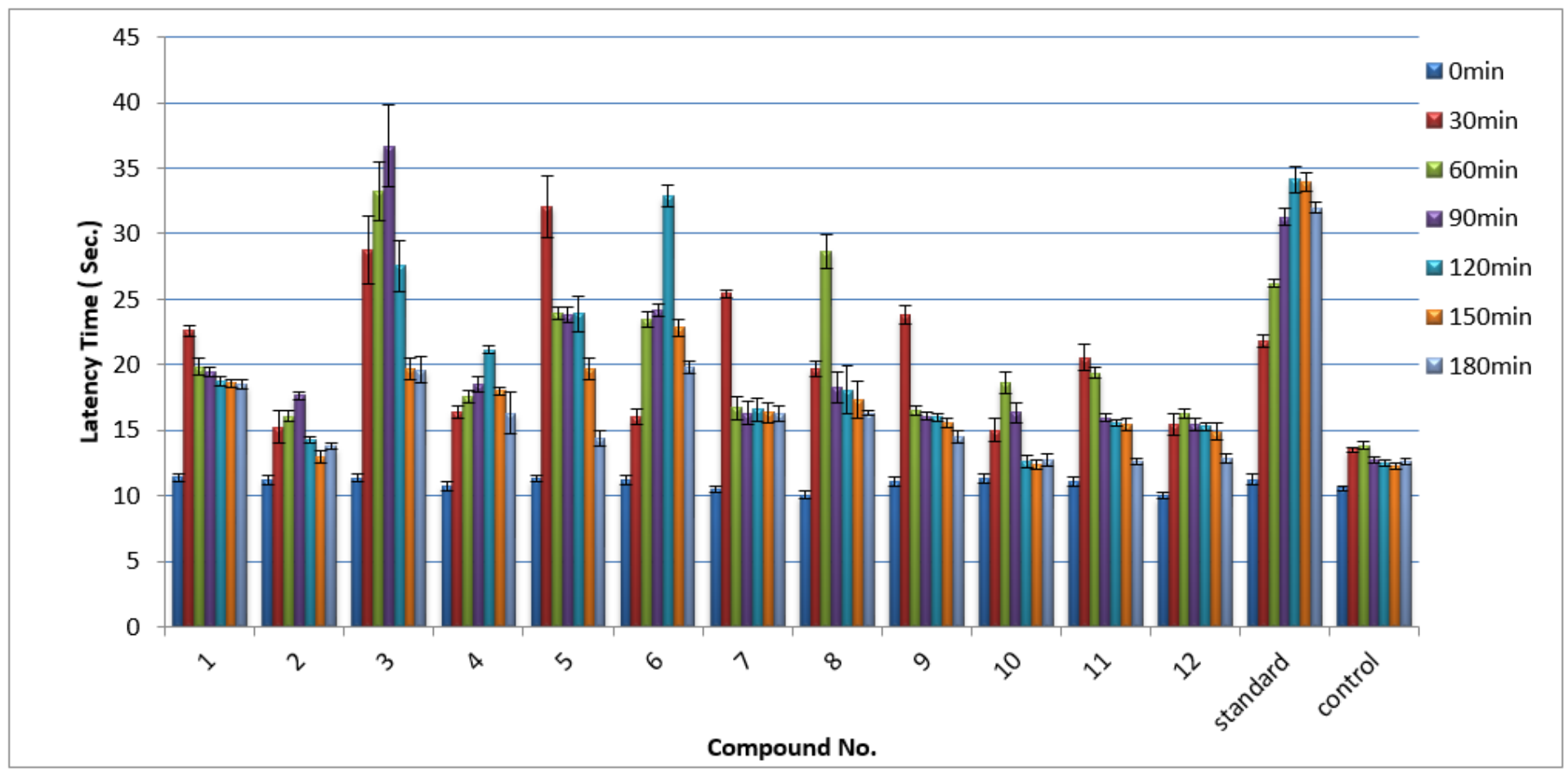

Figure 1

Latency time of TFMP derivatives and morphine sulphate

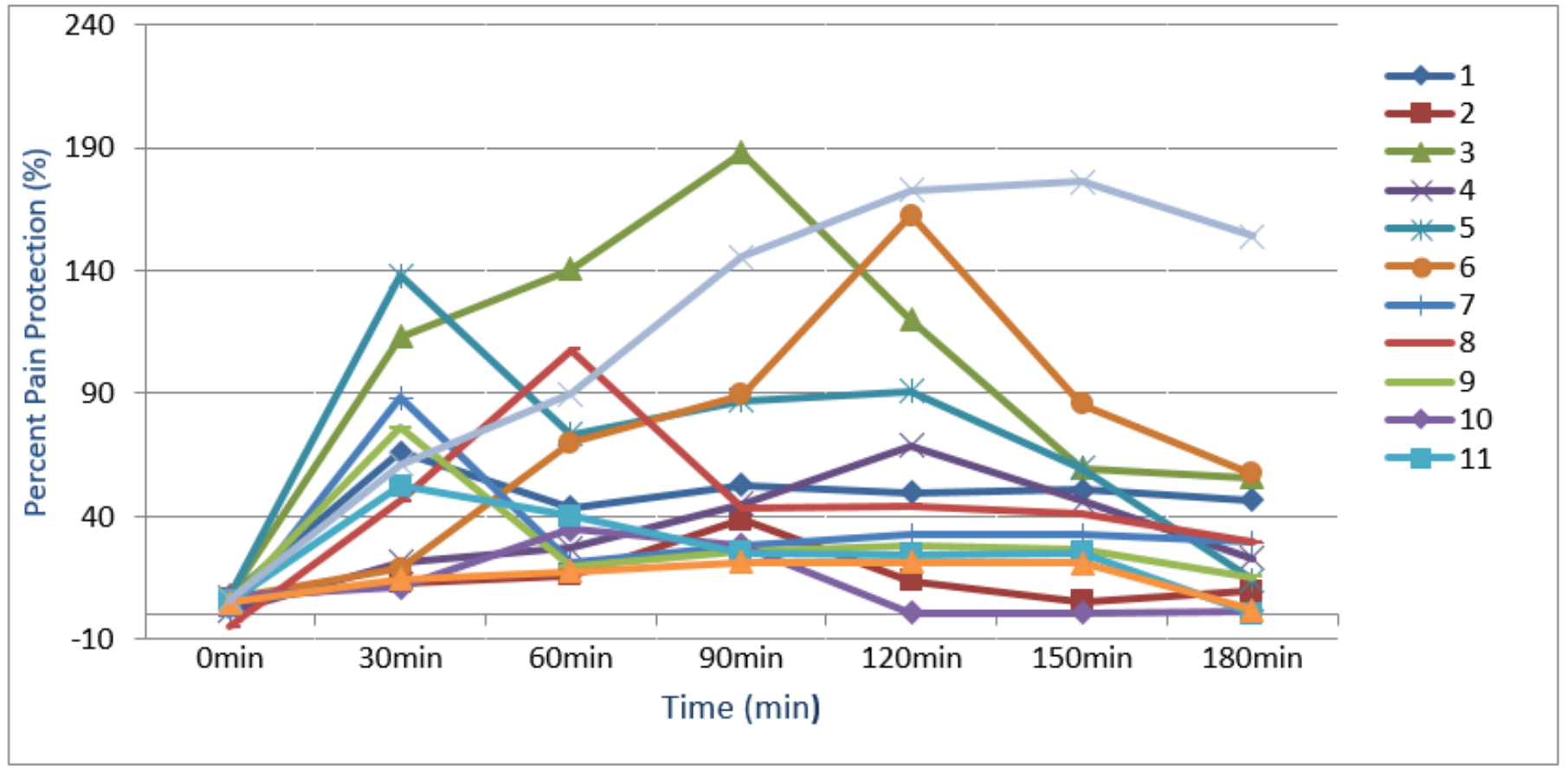

Figure 2 
Percent pain proteciton of TFMP derivatives and Morphine Sulphate

\section{Supplementary Files}

This is a list of supplementary files associated with this preprint. Click to download.

- Scheme.docx

- SupplementaryFileC13NMRnew.doc

- SupplementaryFileHNMRnew.doc

- SupplementaryFileHRMS.doc

- SupplementaryFileMASSSPECTRUMS.doc 